\title{
Stochastic solutions for the Hipparcos astrometric data merging
}

\author{
F. Arenou, L. Lindegren, R. Wielen
}

\begin{abstract}
During the Hipparcos astrometric reduction, an acceptable single or double star solution in agreement with the standard errors of the individual measurements could not be found for some stars. A stochastic model has been applied, which provides an estimate of the intrinsic scatter.
\end{abstract}

\section{Introduction}

The Hipparcos mission has determined accurate astrometric data - 5 parameters: positions, annual proper motions and absolute trigonometric parallaxes - for about 100000 stars. The global data analysis task has been undertaken by two scientific Consortia, FAST and NDAC. They obtained independently two astrometric solutions which were merged in order to produce the Hipparcos Catalogue (118218 entries). A comprehensive description of the mission may be found in [ES97].

Although satisfactory results were obtained for almost all stars, there existed some stars where none of the various models (single star, double star, orbital binary, etc) was adequate, thus leading to a high outlier rejection rate or a high goodness of fit. This may be due for instance to unrecognized duplicity or orbital motion.

Lacking an acceptable deterministic model, one solution could have been to apply robust least-square procedures, which are designed to deal for instance with strongly non-Gaussian tails of the error distribution. In contrast, a stochastic model was assumed for the photocentric displacements superposed on a uniform motion of the concerned stars.

This was implemented by adding quadratically an intrinsic, excess scatter (cosmic error) to the standard errors of all observations. The correlation coefficients between FAST and NDAC observations, calibrated as a function of formal errors, increase with the cosmic error. These coefficients, together with the formal errors, provided the covariance matrix between observations.

For each star, the following procedure was adopted:

1. A normal astrometric solution was performed, still allowing the rejection of exceptional outliers:

(a) A weighted 5-parameter least-square solution was computed, using the individual observations (abscissae). 
(b) The abscissae residuals were computed: the highest residual was rejected if it was greater than 3 times its standard error. If this was not the case, pairs of observations were tested: if the normalized difference was greater than $3 \sqrt{2}$, the pair was rejected.

(c) The procedure was iterated until no outlier or more than 2 outliers was found. If no stochastic solution was found, the maximum number of outliers, 2 , was decreased to 1 , then 0 .

2. Using the non-rejected observations, the cosmic error was computed, together with its standard error. This was done by iteration, finding when possible the value for which the unit-weight variance of the solution was 1 .

3. Until the convergence of the cosmic error, the covariance matrix was computed for a new iteration.

\section{Application}

This method has been applied to all 'single' stars from the Catalogue with a good astrometric solution in order to define a very significant normalized cosmic error (corresponding approximately to a $3 \sigma$ level of a Gaussian twosided test). This criteria has been used to decide if the stochastic solution was retained or not.

Using the orbital binaries in the Catalogue, the cosmic error was found to be clearly correlated with their semi-major axis. This method also allowed the detection of stars with remaining bad input positions, which were found because of their high cosmic error.

The retained stochastic solutions have been applied as a last resort to the stars where all other models failed, 1561 stars of the final Hipparcos Catalogue, of which 643 are suspected doubles. These stars are possibly astrometric binaries with a period of less than a few years, and an estimate of the semi-major axis may then be obtained using the cosmic error found. This would not have been achieved with the blind use of other standard or robust methods.

\section{References}

[ES97] ESA. The Hipparcos Catalogue. ESA SP-1200, Vol I, 1997. 